\title{
Índice cerebro placentario en predicción de resultado perinatal adverso y alteraciones de la frecuencia cardiaca fetal en embarazos no complicados de 40 semanas y mas (1)
}

\author{
Ropacka-Lesiak M, Korbelak T, Świder-Musielak J, Breborowicz G. Cerebroplacental \\ ratio in prediction of adverse perinatal outcome and fetal heart rate disturbances in \\ uncomplicated pregnancy at 40 weeks and beyond. Arch Med Sci 2015;11(1):142-8.
}

Análisis Crítico: Carolina Martinovic Titiro, Jorge A. Carvajal C., PhD.

Unidad de Medicina Materno-Fetal, División de Obstetricia y Ginecología, Escuela de Medicina, Pontificia Universidad Católica de Chile.

\section{RESUMEN (1)}

Introducción: El objetivo del estudio fue determinar la utilidad de la velocimetria doppler, basado en la evaluación del índice cerebro/placentario, en predecir anomalías de la frecuencia cardiaca fetal intraparto y resultado adverso neonatal en embarazos no complicados de 40 semanas y más. Método: 148 mujeres con embarazo no complicados entre 40 y 42 semanas cumplidas, fueron divididas en grupo control y estudio: con ausencia de preservación cerebral (brain-sparing effect) $(n=79)$ y con presencia de preservación cerebral $(n=69)$, respectivamente. El índice de pulsatilidad y resistencia de la arteria cerebral media y umbilical, y el índice cerebro/placentario fueron evaluados diariamente por ultrasonografía doppler. Un índice cerebro/ placentario $<1,1$ fue reportado como sugerente de preservación cerebral. Los índices de flujo anormal fueron analizados y comparados con resultados adversos del embarazo y neonatales. Resultados: En el grupo de índice cerebro/placentario anormal las alteraciones del registro cardiotocográfico fueron significativamente más frecuentes $(62,3 \%)$ que en el grupo de índice cerebro/placentario normal (19\%) $(p=0,0001)$. La comparación de índices de doppler seleccionados revela que el índice cerebro/ placentario muestra la mayor sensibilidad en predicción de alteraciones de la frecuencia cardiaca fetal intraparto $(74,1 \%)$ y resultado neonatal adverso
(87,8\%). Conclusiones: El índice cerebro/placentario muestra alta sensibilidad en predicción de anomalías de la frecuencia cardiaca fetal y resultado neonatal adverso en embarazos no complicados de 40 semanas y más. El índice cerebro/placentario es útil en la práctica clínica en monitorización antenal de estas mujeres, a fin de seleccionar aquellas con alto riesgo de complicaciones intra y post parto.

\section{ANALISIS DE LA INVESTIGACIÓN}

\section{A. Relevancia clínica de la investigación}

La asfixia perinatal se produce por una alteración del intercambio gaseoso a nivel del lecho placentario, activándose mecanismos de glicolisis anaerobia y producción de ácido láctico causando acidosis (2). La asfixia perinatal es causa de morbimortalidad neonatal y secuelas a largo plazo (3). La hipoxia fetal es capaz de activar mecanismos adaptativos, los que entre otras cosas, permiten mantener el flujo de oxígeno a territorios nobles. Entre los territorios nobles cuyo flujo de oxígeno se intenta mantener, pese a la hipoxemia, se encuentran el corazón, el cerebro y las glándulas suprarrenales. La mantención del flujo de oxígeno a estos territorios se logra aumentando el flujo sanguíneo, y el aumento de flujo se logra mediante vasodilatación arterial del territorio protegido, a expensas de vasoconstricción en territorios que no 
se intenta proteger, por ello a este mecanismo de protección se le llama redistribución de flujo (4-5). La redistribución de flujo puede ser clínicamente medida mediante el ultrasonido doppler. En el caso del cerebro, la redistribución de flujo se evidencia como disminución de la resistencia en el doppler de arteria cerebral media, fenómeno que se conoce con el nombre de preservación cerebral (brainsparing effect) (4-5). El índice cerebro/placentario resulta de la división del índice de pulsatibilidad de la arteria cerebral media por el índice de pulsatibilidad de la arteria umbilical, y permite cuantificar la redistribución del gasto cardiaco. Estudios en fetos de corderos han demostrado que el índice cerebro/placentario es el que mejor refleja cambios agudos de la presión de oxígeno. Se ha propuesto que el índice cerebro placentario se altera más precozmente que los índices del doppler de la arteria cerebral media o umbilical por separado, permitiendo así predecir resultados adversos perinatales en fetos con índices de pulsatibilidad y resistencia normal en la arteria umbilical y cerebral media (4-5). El embarazo de postérmino es una condición de riesgo de morbimortalidad fetal/neonatal principalmente en el contexto de hipoxia intrauterina o durante el trabajo de parto. Para evitar los riesgos asociados a la prolongación del embarazo, el tratamiento consiste en la inducción del trabajo de parto. Sería conveniente disponer de un método confiable para predecir el riesgo de morbilidad fetal/neonatal en estos embarazos. Por ello se hace muy relevante esta investigación que pretender responder si el índice cerebro/placentario es un buen predictor de riesgo de hipoxia en embarazos sin complicaciones entre las 40 y 42 semanas.

\section{B. El estudio (1)}

Diseño: Estudio de caso-control realizado en el Departamento de Perinatología de Poznan University of Medical Sciences, Poznan, Poland, entre el 2007 y 2009 que incluyó pacientes embarazadas entre 40 y 42 semanas de gestación. Pacientes: Grupo de estudio, pacientes con hallazgos de centralización de la circulación en el doppler. Grupo control, pacientes sin signos de redistribución de flujo. En total fueron incluidos 148 pacientes cursando un embarazo no complicado entre las 40 y 42 semanas de gestación; 69 pacientes en el grupo de estudio con hallazgos de centralización de la circulación en el doppler y 79 pacientes sin hallazgos de centralización de la circulación en el doppler en el grupo control. Resultados medidos: Correlación y capacidad predictiva entre índices anormales del doppler y resultados fetales y del embarazo. Se analizó específicamente la capacidad predictiva de los índices de arteria umbilical y cerebral media por separado, y el índice cerebro/placentario. Resultado principal medido: Se midieron dos resultados principales: resultado compuesto de patrones anormales del registro cardiotocografico fetal y resultado compuesto de condición adversa del recién nacido. El resultado compuesto de patrones anormales del registro cardiotocografico fetal considera la presencia de cualquiera de las siguientes alteraciones: 1) desaceleraciones variables y tardías, al menos 3 en 30 minutos. 2) variabilidad disminuida o ausente por más de 40 minutos. 3) frecuencia cardiaca fetal basal menor a 110 latidos por minuto o mayor a 150 latidos por minuto por al menos 10 minutos. Condición adversa del recién nacido fue diagnosticada de cumplirse cualquiera de los siguientes criterios: Apgar $<7$ a los 5 minutos, pH de cordón $<7,2$ y exceso de base $<-12 \mathrm{meq} / \mathrm{l}$. Resultados secundarios: Meconio en líquido amniótico, tipo de patología y distrés fetal como causa de cesárea. pH de cordón, exceso de base, presión de $\mathrm{O} 2$, presión de $\mathrm{CO} 2$. Predictores medidos: El predictor analizado fueron los hallazgos de centralización de la circulación en el doppler y un índice cerebro/placentario $<1,1$. Resultados: No hubo diferencias estadísticamente significativas entre el grupo de estudio y el grupo control en términos de edad materna, edad gestacional al parto, tasa de cesárea. El porcentaje de cesárea de emergencia fue significativamente mayor en el grupo con hallazgos de centralización de la circulación en el doppler $(24,6 \%)$ comparado con el grupo control (7,6\%). En el grupo de estudio el porcentaje de registro cardiotocografico anormal $(62,3 \%)$ fue significativamente mayor que en el grupo control (19\%). El estudio revelo que el peso al nacer, Apgar al minuto y a los 5 minutos, $\mathrm{pH}$ de cordón, exceso de base, presión de 02 , presión de $\mathrm{CO} 2$ fue significativamente más bajo en el grupo en las pacientes con hallazgos de centralización de la circulación en el doppler que en el control. Resultados adversos perinatales, recién nacidos pequeños para la edad gestacional, valores de Apgar $<7$ al minuto y a los 5 minutos, presión de $\mathrm{O} 2<15 \mathrm{~mm}$ de $\mathrm{Hg}$, presión de $\mathrm{CO} 2>45 \mathrm{~mm}$ de $\mathrm{Hg}$, $\mathrm{pH}$ de cordón $<7,2$, exceso de base $<-12 \mathrm{meq} / \mathrm{l}$ y meconio en líquido amniótico fueron significativamente mayores en el grupo de estudio versus el grupo control. La alteración en los índices del doppler se asocia significativamente con mayor riesgo de alteraciones en la cardiotocografía y resultado perinatal adverso. La mejor capacidad predictiva fue para el índice cerebro/placentario $<1,1$. Para predecir alteraciones de registro fetal intraparto se evidenció Sensibilidad 74,1\% (IC95\% 0,61-0,85), Especificidad $71,1 \%$ (IC95\% 0,61-0,80), VPP 62,3\% (IC95\% $0,50-0,74)$, VPN $81 \%$ (IC95\% 0,71-0,89) y LR(+) 2,6 . Para predicción de resultado neonatal adverso el índice cerebro/placentario $<1,1$ demostró Sensibilidad 87,8\% (IC95\% 0,74-0,96), Especificidad 68,5\% (IC95\% 0,59-0,77), VPP 51,4\% (IC95\% 0,39-0,64), VPN $93,7 \%($ IC95\% 0,86-0,98) y LR (+) 2,8. 


\section{Análisis crítico}

Validez interna. El diseño del estudio caso control es apropiado para poder establecer un factor predictor o una asociación estadística, de modo que el diseño es apropiado para responder la pregunta del estudio. Sin embargo, no existe cálculo del tamaño muestral, lo que impide saber si los resultados del estudio demuestran una asociación estadística real o solo producto del azar, lesionando la validez interna del estudio. No hubo diferencias demográficas entre ambos grupos estudiados. No se reportaron pérdidas de pacientes. No se informa de los criterios de interrupción del embarazo en ambos grupos ni la existencia de protocolos de inducción, información que es relevante para determinar si existen variables externas que pudieran influir en los resultados obtenidos. No hubo diferencia en la tasa de cesáreas global, pero el grupo estudio si tuvo mayor tasa de cesáreas de emergencia. No se explica si esta mayor tasa de cesáreas se debió a alteraciones en la cardiotocografía, lo que impide detectar relevancia clínica de la investigación. Los resultados adversos neonatales fueron evaluados solo con parámetros agudos, sin analizar otros resultados adversos secundarios como el ingreso a unidad de cuidados intensivos o las complicaciones derivadas de la asfixia, impidiendo evaluar la relevancia clínica de esta investigación.

Validez externa. El estudio podría ser aplicable a nuestra población, sin embargo como los protocolos de seguimiento y momento de interrupción no están especificados tal vez existan factores de manejo clínico que pudieran ser diferentes a los de otras poblaciones. No se explicitan los criterios para efectuar la cesárea de urgencia, impidiendo la comparación con otras poblaciones. La definición de resultado neonatal adverso es diferente a la definición en uso de asfixia perinatal, de modo que los resultados no son comparables con los de otras investigaciones.
Conclusión. Estudio con buen diseño para establecer asociación estadística y predictor de riesgo. Sugiere que el índice cerebro/placentario tiene una alta sensibilidad en predicción de anomalías de la frecuencia cardiaca fetal intraparto y resultado adverso neonatal en embarazos no complicados de 40 semanas y más. Sin embargo, existen graves defectos en el diseño que afectan la validez interna y externa de los datos, haciendo que sus resultados no sean aplicables en el trabajo clínico habitual. Adicionalmente, no se explicita la relevancia clínica de los hallazgos, por lo que la investigación pierde interés clínico.

\section{REFERENCIAS}

1. Ropacka-Lesiak M, Korbelak T, Świder-Musielak J, Breborowicz G. Cerebroplacental ratio in prediction of adverse perinatal outcome and fetal heart rate disturbances in uncomplicated pregnancyat 40 weeks and beyond. Arch Med Sci 2015;11(1):142-8.

2. Shankaran S, Laptook AR, Ehrenkranz RA, Tyson JE, McDonald SA, Donovan EF, et al. Whole-body hypothermia for neonates with hypoxic-ischemic encephalopathy. National Institute of Child Health and Human Development Neonatal Research Network. N Engl J Med 2005;353(15):1574-84.

3. Johnston MV, Trescher WH, Ishida A, Nakajima W. Neurobiology of hypoxic-ischemic injury in the developing brain. Pediatr Res 2001;49(6):735-41.

4. Palacio M, Figueras F, Zamora L, Jiménez JM, Puerto $\mathrm{B}$, Coll $\mathrm{O}$, et al. Reference ranges for umbilical and middle cerebral artery pulsatility index and cerebroplacental ratio in prolonged pregnancies. Ultrasound Obstet Gynecol 2004;24(6):647-53.

5. Baschat A, Gembruch U. The cerebroplacental doppler ratio revisited. Ultrasound ObstetGynecol 2003;21(2):124-7.

6. Gülmezoglu AM, Crowther CA, Middleton P, Heatley E. Induction of labour for improving birth outcomes for women at or beyond term. Cochrane Database Syst Rev. 2012 Jun 13;6:CD004945. 